\title{
Rbpj links uterine transformation and embryo orientation
}

\author{
Cell Research (2014) 24:1031-1032. doi:10.1038/cr.2014.110; published online 22 August 2014
}

Implantation involves complex signaling networks, which direct morphological and molecular transformation of the embryo and the uterus and establish the trajectory of normal pregnancy. The recent work by $Z$ hang et al. published in Cell Research, identifies the transcriptional regulator, Rbpj, as essential for uterine closure and proper embryo alignment during implantation in the mouse, raising the possibility that aberrant Rbpj signaling could contribute to infertility in humans.

Scientists have long appreciated the remarkable precision of embryo orientation during implantation [1]. However, the intricate molecular mechanisms underlying the morphological observations remain unresolved. Due to practical and ethical reasons, it is difficult to study implantation in humans. Therefore, animal models, such as the mouse, are used despite both commonalities and differences between the two species $[2,3]$. For example, the abembryonic trophoblast mediates implantation in the mouse, whereas human embryos implant via the embryonic pole.

Successful implantation requires proper sequencing and coordination of multiple steps, involving the embryo and uterus, that culminate in the establishment of pregnancy [4]. On the embryonic side, they include lineage allocation, trophoblast fate specification, blastocyst hatching from the zona pellucida, and embryo adhesion/invasion. On the uterine side, the steps include establishment of receptivity followed by decidualization, which is propagated from the site of embryo attachment, and luminal closure. The end result is implantation of a properly oriented blastocyst, which depends on these unilateral processes being coordinated in a bilateral manner. Therefore, a great deal of research in this area has focused on the dialogue that coordinates embryonic interactions with uterine transformation. An improved understanding of these processes will be important for optimizing assisted reproductive technologies, including in vitro fertilization (IVF) [5].

The molecular events underlying implantation were recently reviewed $[4,5]$. Hormonal regulators - estrogen and progesterone - delivered at precise levels and times, are required for establishing the window of receptivity. Several signaling pathways play important roles: Lif, Cox2, PPAR $\gamma$, Msx1/2, Sgk1, Ihh, Bmp2, EGF family members, Hox and Wnts. Initiation of implantation is a two-way dialogue, involving receptors and their ligands. For example, initial embryo adhesion involves trophoblast L-selectin interactions with its oligosaccharide ligands carried by MUC1, whose expression is upregulated on the surface of receptive uterine epithelial cells $[6,7]$. The transmembrane receptor, Notch, and its ligands also play critical roles in embryonic-uterine interactions [8]. Additional signaling pathways, including cannabinoid and Wnt, are activated as the embryo becomes competent to implant, termed "blastocyst activation". Further identification of the molecules that promote uterine receptivity and blastocyst activation is critical for understanding the processes involved in implantation. In addition, knowing how novel regulators fit into the current paradigm is important.
Adding to this body of knowledge, the new report by Zhang et al. [9] demonstrates the critical importance of Rbpj in uterine receptivity and embryo implantation. Bound to DNA regulatory regions, Rbpj, the primary transcriptional mediator of Notch signaling, directly interacts with the Notch intracellular domain (NICD) and recruits co-activators, such as MAML, thus providing a mechanism by which Notch signaling induces transcription of target genes [10]. In screening of potential candidate genes involved in peri-implantation uterine transformation, Zhang et al. [9] provide evidence of dynamic Rbpj expression in the mouse uterus during the peri-implantation period. By generating a uterine-specific deletion of $\mathrm{Rbpj}\left(R b p j^{\mathrm{d} / \mathrm{d}}\right)$ via progesterone receptor (PR)-Cre recombination, the authors demonstrate that this molecule is required for implantation. Its absence significantly reduces pregnancy rate and litter size. Detailed morphological analyses revealed abnormal crypts in the uterine lumen rather than the normal vertical slit oriented along a mesometrial-antimesometrial (M-AM) axis, and disorientation of attached embryos. At a molecular level, the authors observed abnormal E-cadherin expression in association with the uterine epithelial cells that lined these crypts. They hypothesize that lodging in these irregular spaces disorients the embryonic axis, which in turn, distorts developmental progression. It will be interesting to investigate the extent to which molecular versus mechanical signals are involved in this phenomenon.

Here, Zhang et al. [9] explore the molecular interactions involved in Rbpj 
regulation of decidual remodeling, luminal closure, and embryonic-uterine orientation. The uterine epithelia of $R b p j^{\mathrm{d} / \mathrm{d}}$ females have a heightened estrogenic response. Co-immunoprecipitation experiments indicate that Rbpj directly interacts with estrogen receptor $\alpha(E R \alpha)$ in the uterus and in a welldifferentiated human endometrial adenocarcinoma cell line (Ishikawa cells). Next, to investigate the relationship between ER $\alpha$ and Rbpj, in the context of Notch signaling, Zhang et al. [9] used two genetically-modified Ishikawa lines - one transfected with Rbpj and the other with Rbpj and DNMAML, the dominant-negative form of MAML and an inhibitor of Rbpj-mediated Notch-dependent signaling. Transfection of Rbpj suppresses ER $\alpha$ responses. However, co-transfection with DNMAML did not reverse these effects. At a mechanistic level, ChIP-PCR failed to demonstrate a direct association between Rbpj and ER $\alpha$ DNA binding affinity. These results suggest that Rbpj disrupts ER $\alpha$ signaling in a Notch-independent manner, implying novel mechanisms of Rpbj repression. Additional experiments using an ER $\alpha$ antagonist in the $R b p j^{\mathrm{d} / \mathrm{d}}$ females further support these observations and demonstrate a specific role for the $\mathrm{Rbpj}$ regulation of $\mathrm{ER} \alpha$ responses in luminal closure and embryonic-uterine orientation, but not in modulating decidual patterning. Additional work will be needed to understand how Rbpj and potentially other molecules with related functions suppress estrogen responses, leading to luminal closure.

In investigating the mechanistic roles of Rbpj in abnormal decidual patterning, Zhang et al. [9] demonstrate that the loss of Rbpj significantly reduces the expression of critical proteinases at the fetal-maternal interface. ChIP analyses identified a regulatory region in the Mmp2 promoter to which Rbpj binds. In contrast to Rbpj and $\mathrm{ER} \alpha$ interactions, transfection of DNMAML in 293T cells reversed the effects of Rbpj on Mmp2 transcription. Additionally, Rbpj regulates the expression of molecules that are important for vasculature patterning and development. Whether this occurs via the Notch pathway remains to be determined.

Altogether, the morphological and mechanistic data presented by Zhang et al. [9], who used in vivo and in vitro models, and multiple experimental strategies, strongly suggest that Rbpj plays critical roles in several aspects of implantation (decidual remodeling, uterine patterning, luminal closure, and vascular development) via Notchdependent and -independent signaling pathways. Further delineating the functional role of Rbpj and other interactors in the uterus will be of great interest as it will establish links to other pathways that regulate implantation and the establishment of pregnancy. Additionally, learning how abnormal uterine architecture disorients embryonic development could yield important insights into the normal process.

Studies such as the paper by Zhang et al. [9], which examines the multifaceted molecular cues that are involved in early pregnancy, suggest a high-level specificity and complexity on both the uterine and embryonic sides. Decoding these interactions may lend insights into why human pregnancy is relatively difficult to achieve. For example, only $30 \%$ of embryos implant during each normal menstrual cycle and $75 \%$ of infertility cases are attributable to implantation failure. The impact at a population level is enormous as $\sim 15 \%$ of couples are infertile. Future studies will need to address how molecular interactions that initiate pregnancy in the mouse, such as those regulated by Rbpj, correspond to parallel processes in human. In addition, it will be interesting to explore how a combination of environmental and genetic factors may disrupt the finely-tuned dialogue that is required for normal implantation and the initial stages of embryonic development.

Joshua F Robinson ${ }^{1,2,3}$, Susan J Fisher ${ }^{1,2,3,4,5,6}$

${ }^{I}$ Center for Reproductive Sciences, ${ }^{2}$ Department of Obstetrics, Gynecology, and Reproductive Sciences, ${ }^{3}$ The Eli \& Edythe Broad Center for Regeneration Medicine and Stem Cell Research, ${ }^{4}$ Division of Maternal Fetal Medicine, ${ }^{5}$ Department of Anatomy, ${ }^{6}$ Human Embryonic Stem Cell Program, UCSF, San Francisco, CA 94143, USA Correspondence: Susan J Fisher

E-mail: sfisher@cgl.ucsf.edu

\section{References}

1 Chen Q, Zhang Y, Elad D, et al. Mol Aspects Med 2013; 34:1024-1042.

2 Bazer FW, Spencer TE, Johnson GA, et al. Reproduction 2009; 138:195-209.

3 Lee KY, DeMayo FJ. Reproduction 2004; 128:679-695.

4 Cha J, Sun X, Dey SK. Nat Med 2012; 18:1754-1767.

5 Koot YE, Teklenburg G, Salker MS, et al. Biochim Biophys Acta 2012; 1822:19431950.

6 Carson DD, Julian J, Lessey BA, et al. Front Biosci 2006; 11:2903-2908.

7 Genbacev OD, Prakobphol A, Foulk RA, et al. Science 2003; 299:405-408.

8 Cuman C, Menkhorst E, Winship A, et al. Reproduction 2014; 147:R75-R86.

9 Zhang S, Kong S, Wang B, et al. Cell Res 2014; 24:925-942.

10 Tanigaki K, Honjo T. Curr Top Dev Biol 2010; 92:231-252. 